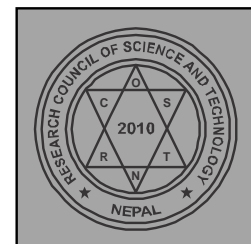

\title{
BIBECHANA
}

A Multidisciplinary Journal of Science, Technology and Mathematics

ISSN 2091-0762 (online)

Journal homepage: http://nepjol.info/index.php/BIBECHANA

\section{Electronic structure and magnetic properties of bulk elements (Fe and Pd) and ordered binary alloys (FePd and Fe3Pd):TB-LMTO-ASA approach}

\author{
Suraj Pandey ${ }^{1}$, Gopi Chandra Kaphle ${ }^{2}$, Narayan Prasad Adhikari ${ }^{2 *}$ \\ ${ }^{1}$ Patan Multiple Campus, Patan Dhoka, Kathmandu, Nepal \\ ${ }^{2}$ Central Department of Physics Tribhuvan University, Kathmandu, Nepal \\ *Email: npadhikari@gmail.com
}

Accepted for publication: February 05, 2014

\begin{abstract}
The binary alloys formed from the elements of Fe and Pd like FePd and Fe3Pd, have the strange electronic and magnetic properties, generally used in shape memory alloys, Computational chips, Medical purposes as well as modern technological uses. We used TB-LMTO-ASA Approach for the study of band structure and density of states of the transitional elements $\mathrm{Fe}$ and Pd and their ordered alloys FePd and Fe3Pd. We analyzed metallic and nonmetallic character and occupation of $s, p$ and $d$ electrons from the band structures and magnetic properties through the plot of density of states. Our findings show that all the elements and alloys bear metallic nature whereas bulk Palladium shows non magnetic behavior. Similarly Fe and both the ordered alloys FePd and Fe3Pd are found to be magnetic in nature with values $2.19 \mu_{\mathrm{B}}, 3.49 \mu_{\mathrm{B}}$ and $3.01 \mu_{\mathrm{B}}$ respectively. These well agree with experimental as well as previous theoretical calculations.
\end{abstract}

(C) 2014 RCOST: All rights reserved.

Keywords: Binary alloys; Density of states; Electronic properties; TB-LMTO-ASA.

\section{Introduction}

The physical properties of metal are generally determined through its electronic structure. In solid there are large numbers of electrons and nuclei which are mutually interact with each other, so dynamics of these particles, in general cannot be considered separately. For the simplicity, we have considered frozen core approximation because the nuclei together with core electrons do not take part in bonding. The cohesive, electronic, optical, magnetic and superconducting properties of solids are dominated by the behavior of valance electrons moving in the field of the ion-cores of constituent atoms [1].

Alloys are metallic materials consisting of two or more elements combined in such a way that they cannot be readily separated by physicals means. If the alloy is formed by a mixture of only two types of atoms, it is called binary alloy [2]. Binary alloys may be ordered or disordered depending upon atomic positions. In ordered alloys atoms are situated by making symmetry in the crystals whereas in disordered alloys atoms are situated randomly in the crystals without any crystal symmetry. In the present work we are dealing with alloys and we are also familiar that more than 90 percent of metals used are in the form of alloys. They represent an enormous family of engineering materials that provide a wide range of products with 
S. Pandey et al. / BIBECHANA 11(1) (2014) 60-69: (Online Publication: March, 2014) p.61

useful properties. Each alloy is distinct from its components, and the properties of each alloy are distinct. Indeed the purpose of forming an alloy is to provide a metallic substance with physical, mechanical and chemical properties and characteristics that are different from those of its components. Fe3Pd alloys are potentially useful ferromagnetic shape memory materials with a maximum predicted strain of up to $6.8 \%$. Shape memory materials have found widespread application in engineering and medicine, and recently there has been a great deal of interest in the search for ferromagnetic shape memory alloys capable of large reversible strains under an applied magnetic field [3]. FePd alloys have large magnetization and magneto-crystalline anisotropies and as such are potentially technologically important for applications as permanent magnets or high-density storage media [4]. The large number of experimental and theoretical works have been done regarding the characteristics of Fe and Pd separately and their alloys.

The rest of the paper is organized as follows. Section II describes the computational details of the present study. Results and discussions are described in section III whereas section IV provides the conclusion and finally we present references used in the present study.

\section{Computational details}

All the systems considered are studied using Tight Binding Linear Muffin-Tin Orbitals Atomic Sphere Approximation (TB-LMTO-ASA). The calculations are carried out from self-consistent calculations (SCF) based on density functional theory within the framework of the local-density approximation (LDA) [5]. The SCF solve the Kohn-Sham equation using the exchange-correlation potential of Von Barth and Hedin [6] with the help of minimal basis sets and partial wave method implemented in TB-LMTO-ASA code. The crystal potential is constructed of overlapping Wigner-Seitz spheres for each atom in the unit cell. According to the spirit of the TB-LMTO-ASA procedure, only the energetically higher-lying valence states have been included in the self-consistent calculations of the effective crystal potential [7]. In such a case, the deeper lying core states are treated as atomic like and hence called frozen core approximation. The band calculation technique are divided into two main approaches; one uses trial wave function, which is formed as linear combinations of basis functions like plane waves in the nearly free-electron (NFE) method expands orthogonalized plane waves function into a set of energy-dependent partial waves and applies a matching condition for partial waves at the muffin-tin sphere like the APW [8] and KKR methods [9]. Actually, the linearized muffin-tin orbital (LMTO) method developed by Anderson [10,11] uses as basis set for expanding the wave function, a set of orbitals that form a complete basis set for a muffin-tin potential.

For the calculation of $\mathrm{Fe}(\mathrm{BCC})$ and $\mathrm{Pd}(\mathrm{FCC})$ structure same basis set were used at which $(8 \times 8 \times 8) \mathrm{k}$ points were sampled in the irreducible wedge of Brillouin zone (BZ). These parameters are sufficient in leading to well converged total energy and other related parameters. The magnetic moment was calculated by differentiating up and down DOS. After the convergence of SCF we analyzed all the data required for band structure, total energy, DOS etc. All the calculations were iterated to self-consistency with accuracy of $10^{-6}$ Rydberg.

\section{Results and discussions}

This section contains the result and discussion of main findings for the band structure and density of states of elemental solid Fe, Pd and the ordered alloys formed by them like FePd and $\mathrm{Fe}_{3} \mathrm{Pd}$.

\section{A. Band Structure of Fe and Pd}

Presently, we used BCC structure of Iron which belongs to the space group Im-3m with space group number 229. The energy minimization curve for Fe and Pd are shown in Fig. 1. 
S. Pandey et al. / BIBECHANA 11(1) (2014) 60-69: (Online Publication: March, 2014) p.62
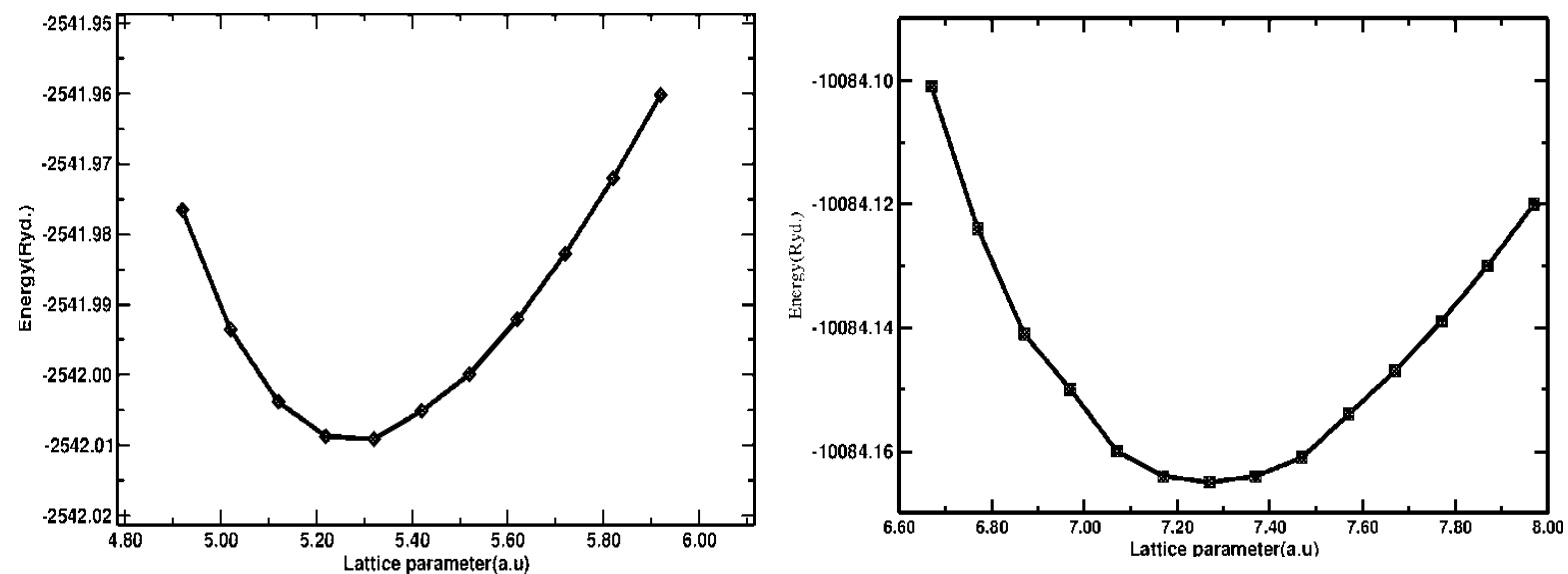

Figure 1: Plot of energy versus lattice constant for Fe (left) and Pd (right).

From the left panel of Fig. (1) we found that the lattice parameter of Fe at minimum energy is $2.83 \stackrel{\circ}{\AA}$ (5.34 a.u.). This value of lattice parameter is $1.39 \%$ less than the experimental value $2.87 \stackrel{\circ}{\mathrm{A}}$ [12]. In case of Palladium we have used FCC structure which belongs to the space group Fm-3m. The energy minimization curve for it is shown in right panel of Fig. (1). We found out that lattice parameter of Pd at minimum energy is $3.86 \stackrel{\circ}{\AA}$ (7.28 a.u.), this value of lattice parameter is $0.77 \%$ less than the experimental value $3.89 \stackrel{\circ}{\mathrm{A}}[12]$. The band structures and density of states of these clusters are calculated with the base of these parameters.
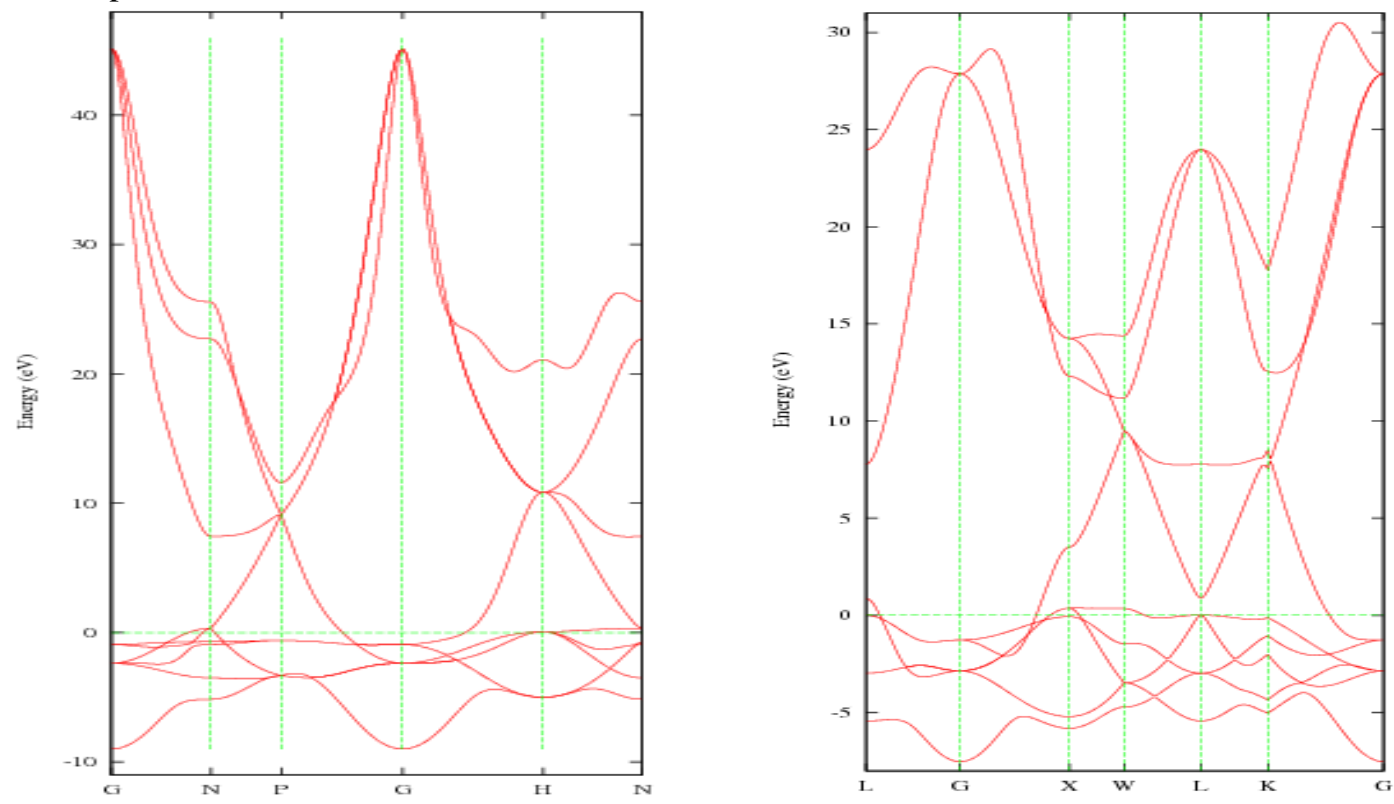

Figure 2: Energy band structures of $\mathrm{Fe}$ and $\mathrm{Pd}$ along symmetry axes with reference to the Fermi energy.

The band structure of Fe and Pd are shown in left panel and right panel of Fig. (2) respectively, which are plotted with reference to the Fermi energy, 0 on vertical axis represents Fermi energy of the iron. Bands below the Fermi energy are valance bands and bands above the Fermi energy are conduction bands. 
S. Pandey et al. / BIBECHANA 11(1) (2014) 60-69: (Online Publication: March, 2014) p.63

In case of Fe there are all together 9 bands. The maximum energy is at conduction band having value $45.36 \mathrm{eV}$ and minimum value of the energy lies at valence band which has the value $-8.67 \mathrm{eV}$. The difference between maximum value of energy of conduction band and minimum value of energy in valance band is found to be $54.01 \mathrm{eV}$. Conduction band and valance bands are overlapping within this range showing $\mathrm{Fe}$ as a metallic nature. Due to the high symmetry of the crystal at the maximum energy of the conduction band and minimum value of energy of the valance band occurs at the Gamma point.

Fat band structure calculations were carried out in order to know the s, p and d orbital contributions on the band structure of Fe. Fig. (3) shows the contribution of $s$ and p orbitals on the band structure of Fe.
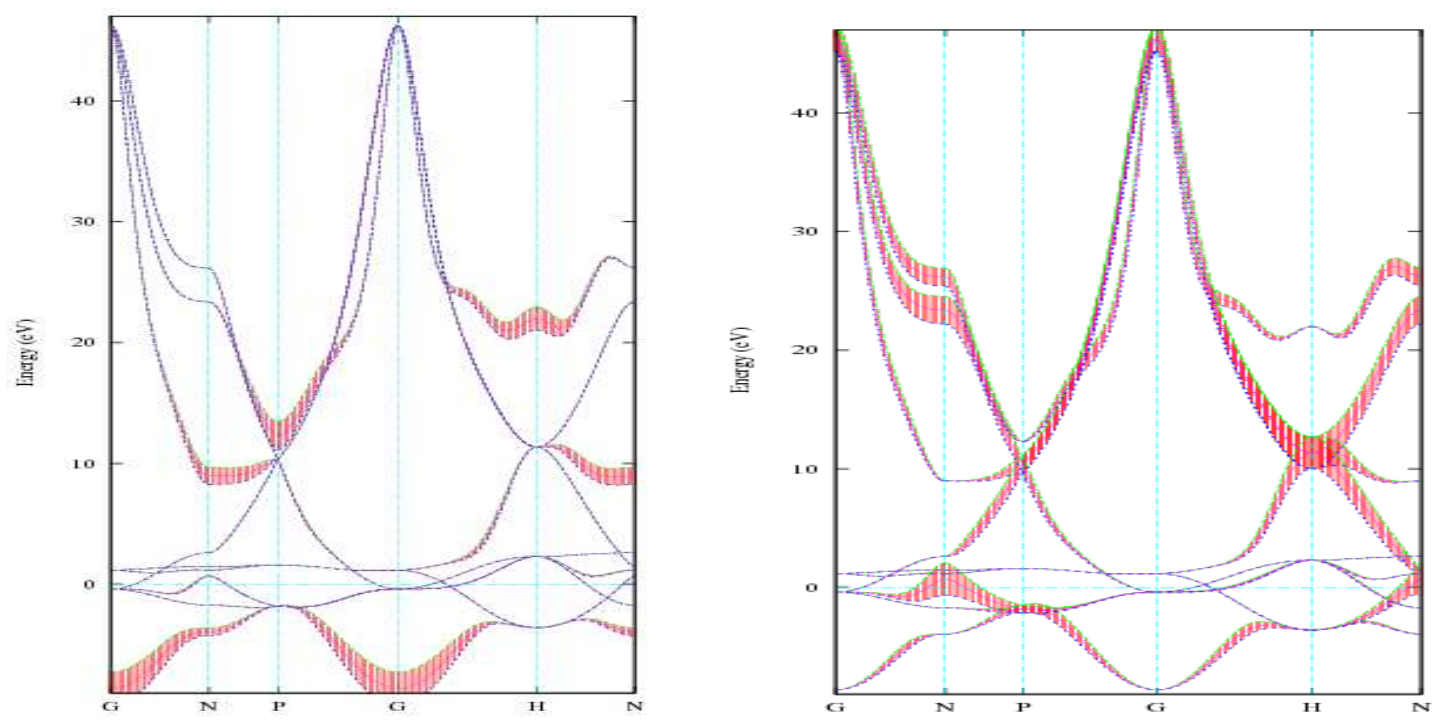

Figure 3: Fat band structure of s-orbital (left) and p-orbital (right) of Fe, horizontal dotted line represents the fermi level.
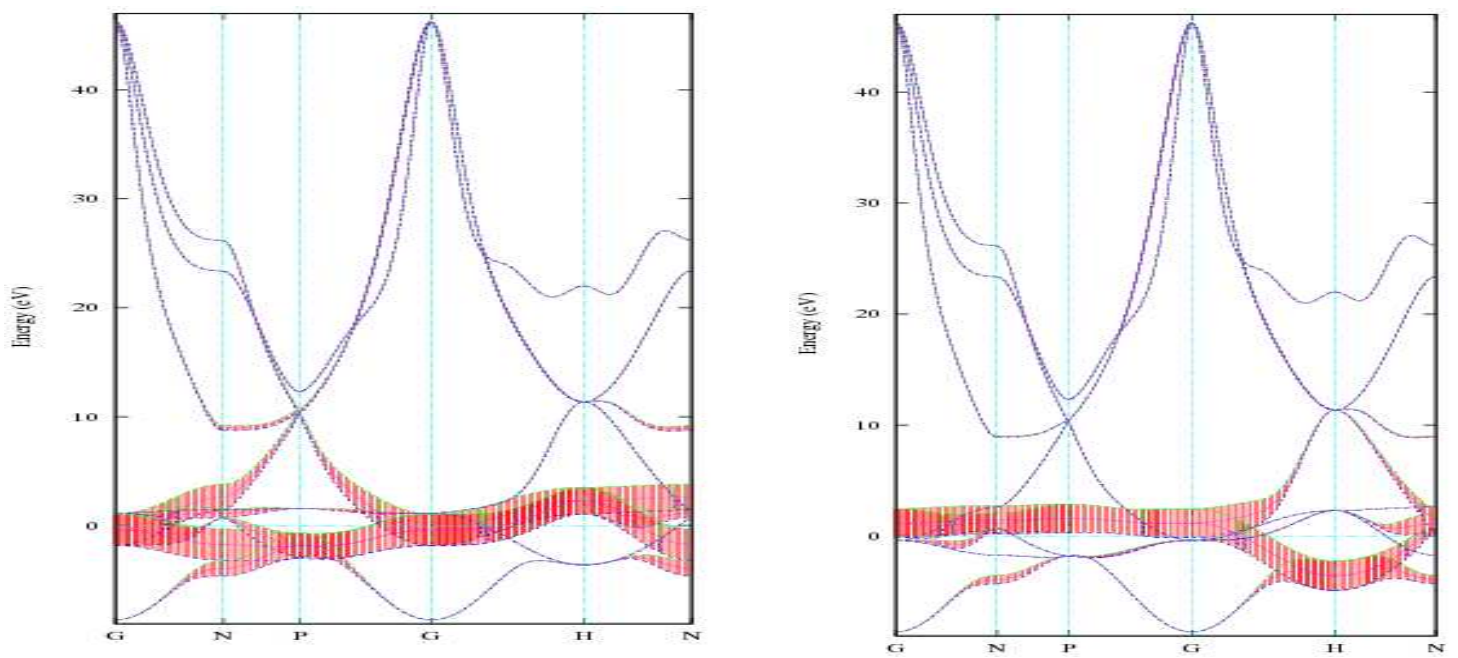

Figure 4: Fat band structure of t2g-orbital (left) and eg-orbital (right) of Fe, horizontal dotted line represents the fermi level. 
It shows that $\mathrm{s}$ and $\mathrm{p}$-orbitals do not contribute much in the band structure of $\mathrm{Fe}$, there is a greater contribution of p-orbital to the conduction band in comparison to the valence band, while the contribution of s-orbital is negligible in both conduction and valence band. $\mathrm{S}$ band contribution for the electronic structure concerntrayed as the $\mathrm{G}$ points . The contribution of $\mathrm{P}$ band elongates from $\mathrm{N}$ to $\mathrm{P}$ and crosses Fermi energy through N. Figure (4) shows that d-orbitals overlap between the valence bands and conduction band at the fermi energy level, it has a greater occupancy at valence band and less at conduction band, this indicate that d-orbital contributes significantly to the total band structure. It is mainly due to majority of electrons are in d-orbitals of Iron.

In case of Pd, valance bands lie below the Fermi energy are found occupied, bands above the Fermi energy are unoccupied conduction band. Valance bands and conduction bands overlaps with each other. Along $\mathrm{G}$ axis maximum value of energy of conduction band is $28.17 \mathrm{eV}$ and minimum value of the energy of valance band is $-7.72 \mathrm{eV}$; difference between these two energies is $35.89 \mathrm{eV}$. Maximum value of energy of conduction band is $30.45 \mathrm{eV}$. Fig. (6) shows that energy levels at some points are degenerate.
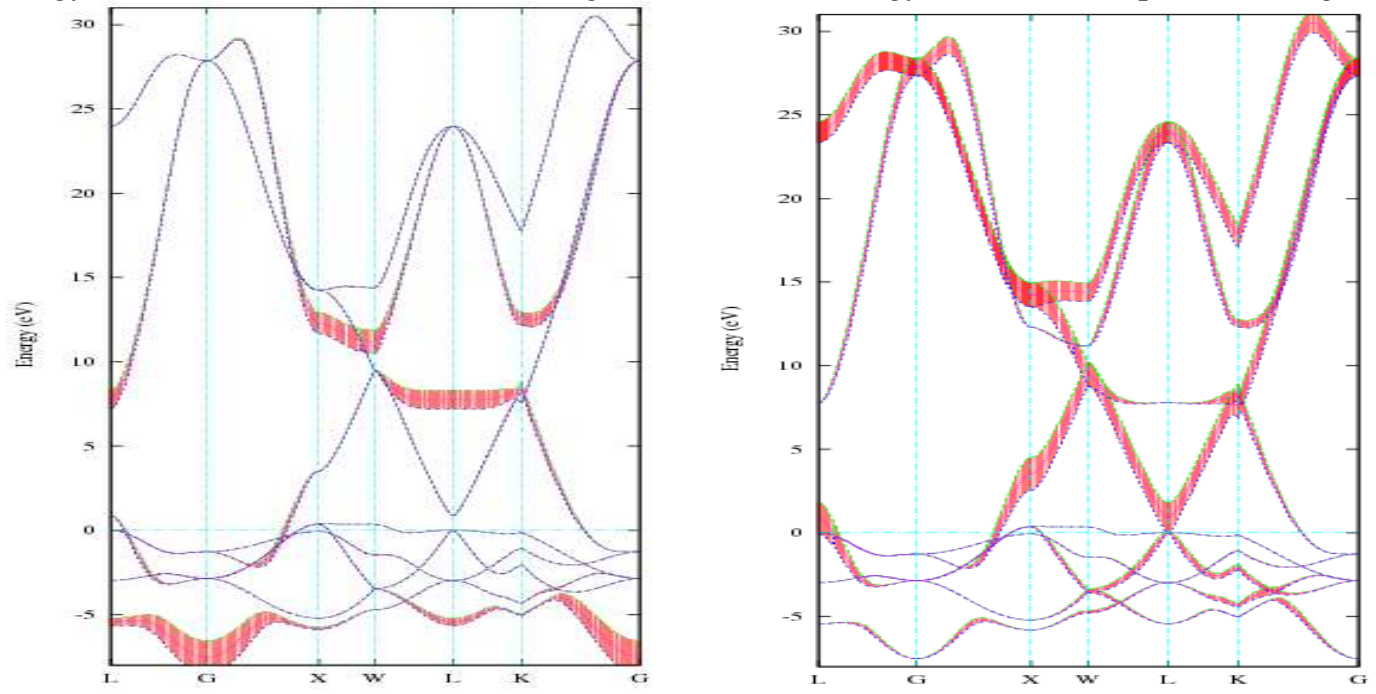

Figure 5: Fat band structure of s-orbital (left) and p-orbital (right) of Pd, horizontal dotted line represents the fermi level.
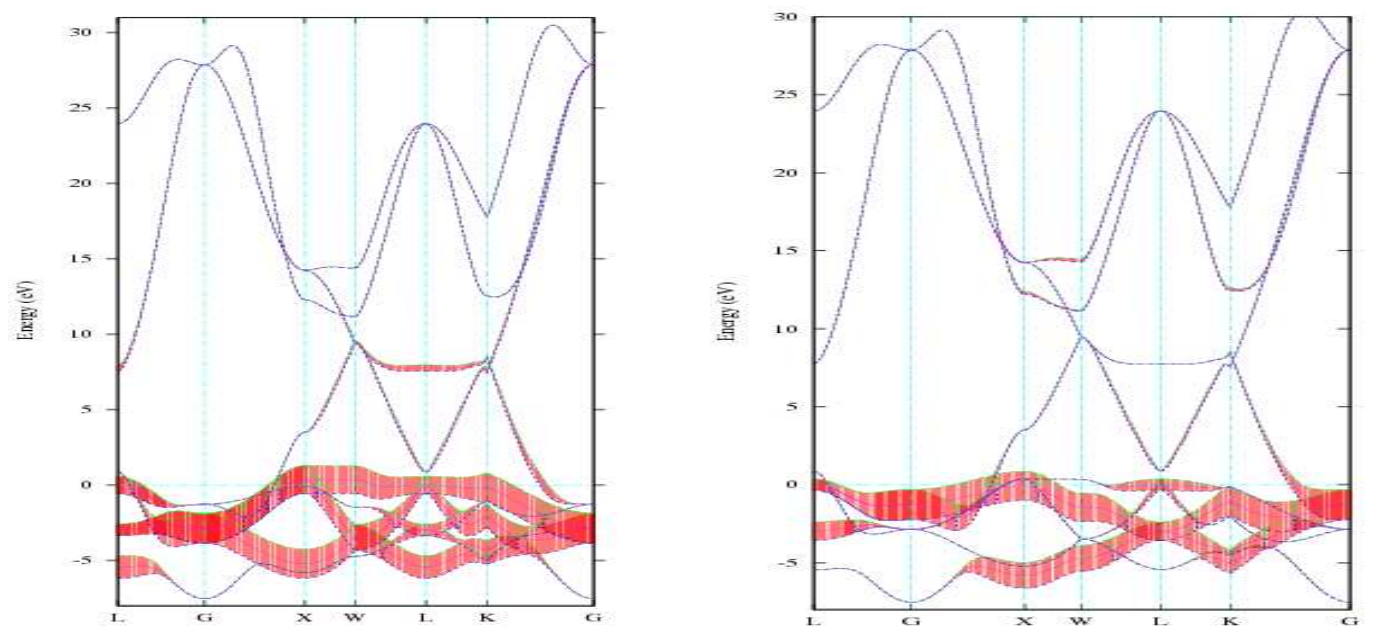

Figure 6: Fat band structure of $\mathrm{t} 2 \mathrm{~g}$-orbital(left) and eg-orbital (right) of Pd, horizontal dotted line represents the fermi level. 
S. Pandey et al. / BIBECHANA 11(1) (2014) 60-69: (Online Publication: March, 2014) p.65

We studied the fat band structures of $s, \mathrm{p}$ and $\mathrm{d}$ orbitals of $\mathrm{Pd}$ in order to know their contributions on the band structure. Dark and shaded portion of Fig. (5) shows the occupancy of s and p electron. Occupancy of the s-orbital is negligible in comparison with the p orbital, occupancy of p-orbital is greater at conduction band and less at the valence band. Fig. (6) shows that the occupancy of the d-orbital occurs at the valence band, while its occupancy at the conduction band is almost negligible due to which it has a significant contribution to the band structure. Physical properties of the solid such as electrical and magnetic properties, is contributed due to the d-orbital.

\section{B. Band Structure of FePd and Fe3Pd}

We deal with the ordered structure of the $\mathrm{FePd}$ and $\mathrm{Fe}_{3} \mathrm{Pd}$ alloys, both have a simple cubic structure with the space group Pm-3m ( Space group no. 221)[13-15]. In the present calculation
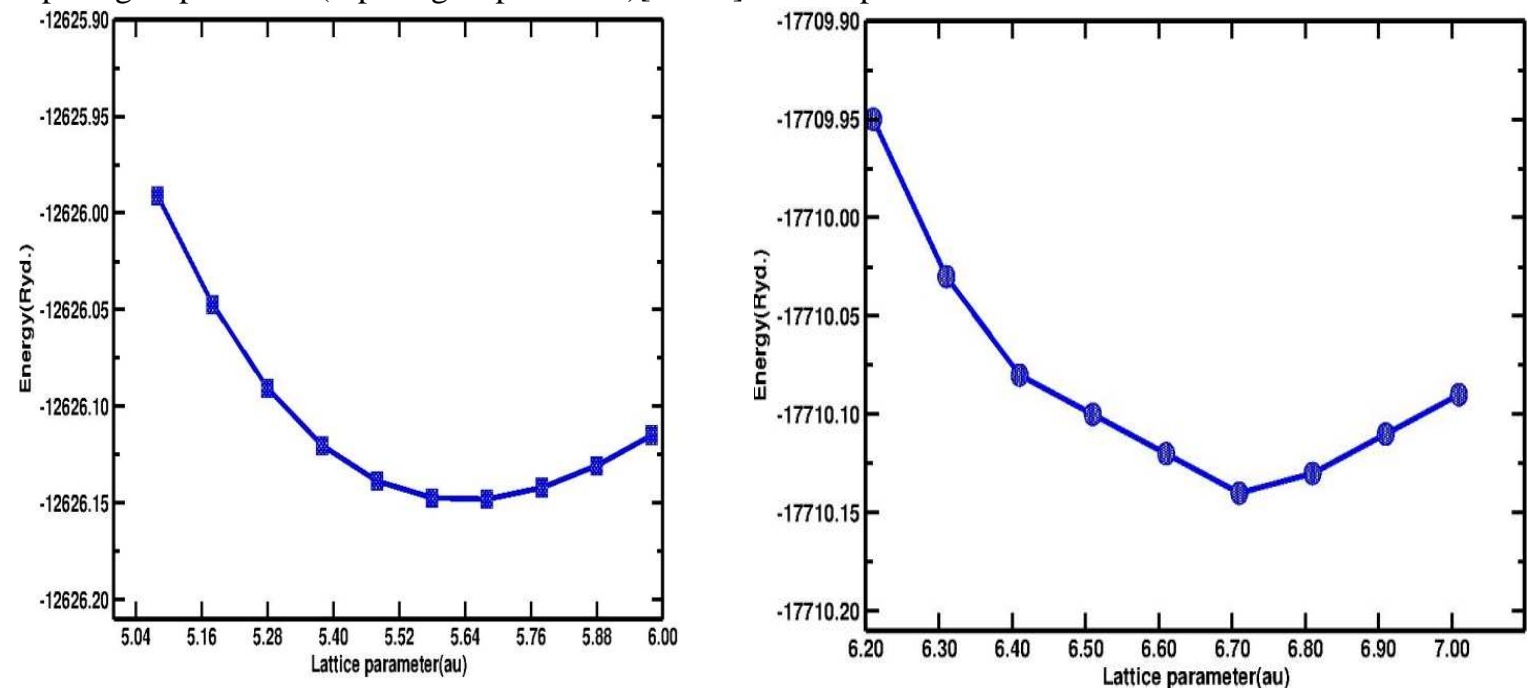

Figure 7: Plot of Energy vs lattice constant for FePd and Fe3Pd.
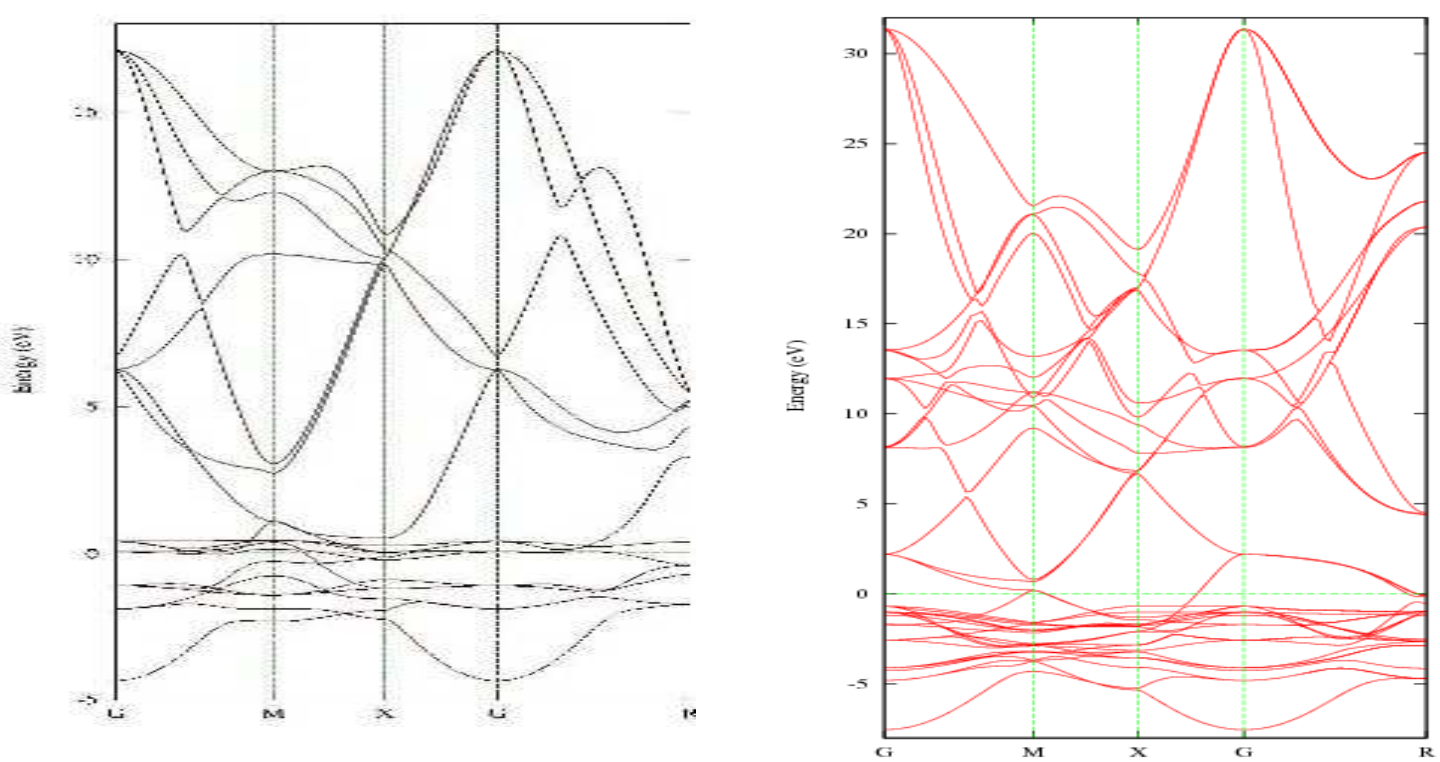

Figure 8: Band structure of simple cubic FePd and Fe3Pd along symmetry axes with reference to the Fermi energy. 
the lattice constant of FePd for the minimum energy is obtained as $3.01 \mathrm{~A}$ (5.68 a.u.). Similarly the lattice parameter for the lowest energy value of $\mathrm{Fe}_{3} \mathrm{Pd}$ is found to be $3.56 \stackrel{\circ}{\AA}$ (6.70 a.u.). The energy minimization curves for both $\mathrm{FePd}$ and $\mathrm{Fe}_{3} \mathrm{Pd}$ are shown in Fig. (7). All the further calculations are done using these optimized lattice parameters.

The band structure of FePd and Fe3Pd obtained from the present study is shown in the left and right panel of Fig. (8). In case of FePd, we found 18 bands with valence and conduction bands overlapping with each other, showing FePd has metallic nature. The minimum value of energy of valence band is $-4.25 \mathrm{eV}$, and the maximum value of energy of conduction band is $21.25 \mathrm{eV}$, at $\mathrm{G}$ point. Similarly, In case of $\mathrm{Fe}_{3} \mathrm{Pd}$ we found 36 bands contributing s, p and d orbitals. The valence and conduction bands also found to be overlapped with each other showing metallic nature of $\mathrm{Fe}_{3} \mathrm{Pd}$ alloy. The maximum energy of the conduction band lies at $\mathrm{G}$ point having value $31.36 \mathrm{eV}$ and minimum energy of valence band at $\mathrm{G}$ point which has a value of $-6.72 \mathrm{eV}$. To know the effective contribution of individual orbital we have to analyze density of states.

\section{Density of States for Fe and Pd}

The plots of total density of states and partial density of states for $s, p$ and d orbitals calculated using optimized parameters for Fe and Pd are shown in left and right panel of Fig. (9).
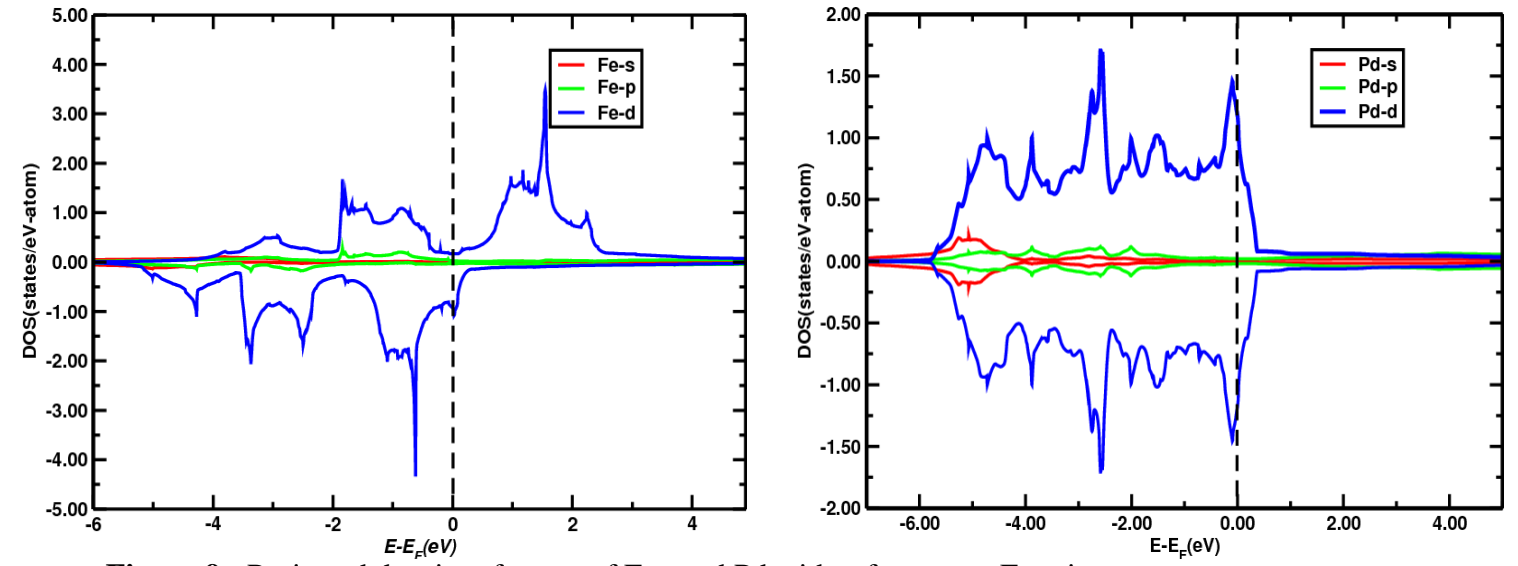

Figure 9: Projected density of states of Fe and Pd with reference to Fermi energy.

From the figure it is found that there is negligible contribution of $\mathrm{s}$ band for the magnetization whereas slight asymmetric nature of $\mathrm{p}$ orbital below the fermi level shows that $\mathrm{p}$ contributed less. There is great asymmetry in up and down spin of DOS of d orbitals indicating that overall magnetic behaviour of Fe is dominated by d electrons. The vertical dotted line at zero represents Fermi energy. The large number of peaks lies below the Fermi energy and few peaks lie above the Fermi energy in left panel of Fig. (9). The peaks in the DOS indicate the large number of states at the certain energy level, which can be occupied by large number of electrons. The magnetic moments are found by integrating the difference of density of states between up and down spin states up to Fermi energy. From the present calculation magnetic moment of $\mathrm{Fe}$ found to be equal to $2.19 \mu_{\mathrm{B}}$ which is $1.35 \%$ less than the experimental $2.22 \mu_{\mathrm{B}}$ obtained by W. Zhong et al. [16] which shows that Fe is strongly ferromagnetic. Partial density of state plot for the $\mathrm{s}, \mathrm{p}$ and $\mathrm{d}$ orbital of Fe shows that there is a significant difference between the up and down spin density of state plot of the d-orbital which give rise to a net magnetic moment.

In case of Pd, we found up spins and down spins density of states are nearly symmetrical with each other due to which the net magnetic moment of Pd is nearly zero, hence Pd is non-magnetic in nature. Partial density of state plot for $\mathrm{s}$ and $\mathrm{p}$ orbital shows that it has a minimum difference of energy between up and down spin hence it does not contributes to the magnetic properties while in case of d-orbital up and down spin DOS curves are almost symmetrical and there is no any significant differences of energy in the density of state plot and hence contribution to the magnetic moment is almost negligible. This supports the arguments given by band structure plot. 


\section{Density of states of FePd and Fe3Pd.}

We have performed calculation of density of states of ordered binary alloy FePd and Fe3Pd. Fig. (10) shows the total density of state plot of ordered binary alloy FePd. All the up and down spin density of states of FePd are plotted with reference to the Fermi energy at which vertical line at zero in the figure represents Fermi energy of the system. Density of states on positive side of the vertical axis are for up spin, and on negative side are for down spin states of FePd. Fig. (10) shows that there is large number of peaks lies below the Fermi energy and few peaks lie above the Fermi energy for the up spin. The highest peak of Up spins lies above the Fermi energy and Down spins lies below the Fermi energy.
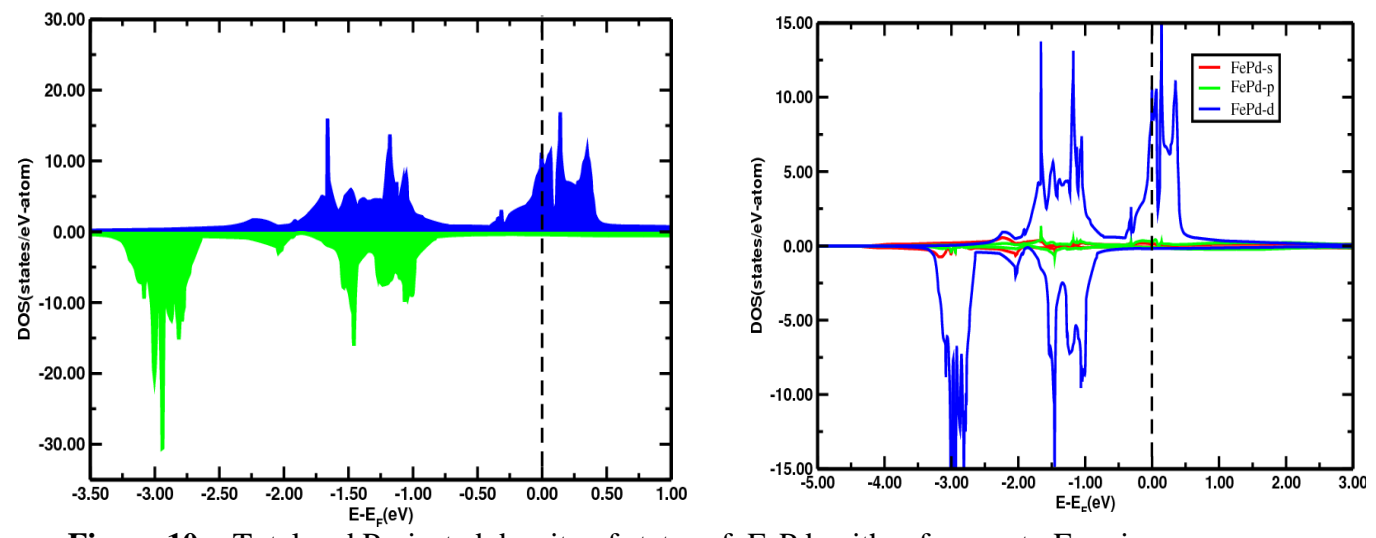

Figure 10: Total and Projected density of states of FePd with reference to Fermi energy.

The DOS of down spin shows that the curve above the Fermi energy is almost smooth having no peaks while below the fermi level it has a large no of peaks. There is a significant differences between the up and down spin DOS in FePd which results in the large magnetic moment. From data obtained for density of states of FePd, the d magnetic moment of FePd was found to be $3.49 \mu_{B}$. The density of state plot for s and p-orbital shows that it has a minimum difference of energy between up and down spin hence it does not contributes to the magnetic moment while there is a significant differences between the up and down spin in the DOS of d-orbitals indicating that magnetic moment mostly come from the contribution of $d$ electrons.

Total density of states of the binary ordered alloy Fe3Pd from the experimental work and present calculation are projected in left and right panel of Fig. (11) respectively. From the right panel of Fig. (11) we found that there is large number of peaks lie below the Fermi energy and few peaks lie above the Fermi energy. The highest peaks of up spins as well as down spins are below the Fermi energy. The significant differences between the up and down spin density of state in Fe3Pd up to the Fermi energy gives the magnetic moment which is found to be equal to $3.01 \mu \mathrm{B} . \mathrm{S}$ and $\mathrm{P}$ contributions are almost negligible. In case of up spin density of state plot of d-orbital has large number of peak lying below the Fermi energy and the curve is almost smooth above the Fermi energy without any peaks. Down spin density of state plot of d-orbital shows that number of peaks below the Fermi energy is greater than above the Fermi energy. Every peaks and nature of the present calculation is exactly matches with the experimental obtained DOS by R. A Stern et al. [3] shown in left panel of Fig. (11). The slight difference in number of states is due to the effect of approximation used in theory and constrained used to experimental works like pressure, temperature etc. From this we considered that overall that DOS is dominated by d projected DOS in Fe3Pd and hence reflect same behaviour to magnetic moments. 
S. Pandey et al. / BIBECHANA 11(1) (2014) 60-69: (Online Publication: March, 2014) p.68
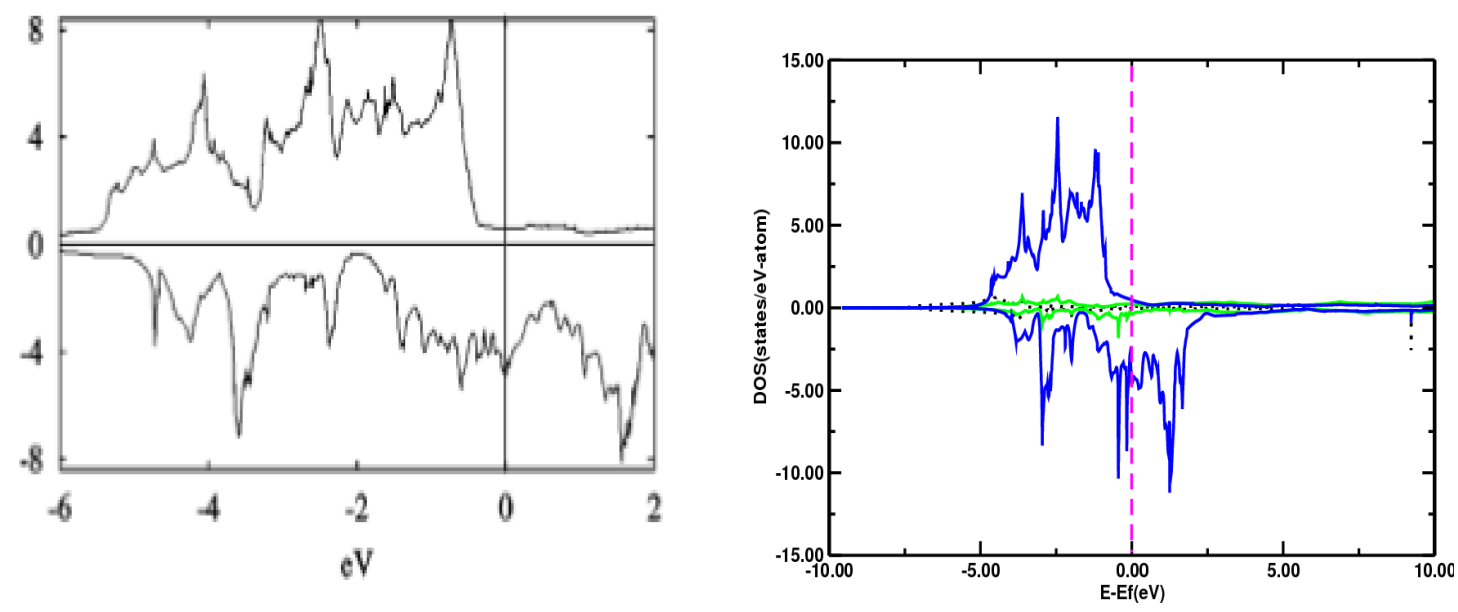

Figure 11: TDOS (Ref:3) and PDOS (present calculation) of Fe3Pd with reference to Fermi energy.

\section{Conclusions and concluding remarks}

The present work gives the calculation of band structure and density of states of elemental solids $\mathrm{Fe}$ and $\mathrm{Pd}$ and ordered binary alloy $\mathrm{FePd}$ and $\mathrm{Fe} 3 \mathrm{Pd}$ using TB-LMTO-ASA approach. In all elemental solids $\mathrm{Fe}$ and Pd along some symmetrical axis we have found nine bands with valance and conduction bands overlapping with each other. This is due to the contribution of minimal basis set used LMTO. In case of ordered binary alloys $\mathrm{FePd}$ and $\mathrm{Fe}_{3} \mathrm{Pd}$ we have found 18 and 36 bands respectively with valance and conduction bands overlapping with each other. This shows that all the elements and their binary alloys behave metallic nature.

The band structures and DOS plot show that the transition metals have partially filled d-shells and their dbands extend through the Fermi surface. Since the d-bands are narrow and contain more levels than free electron bands, the density of states at the Fermi level become very high. The magnetic moment is due to asymmetric distribution of $\mathrm{d}$ bands in the fermi level. The density of states of up and down spins for $\mathrm{Fe}$, $\mathrm{FePd}$ and $\mathrm{Fe}_{3} \mathrm{Pd}$ are found to be quite asymmetric in nature which causes a net magnetic moment and thus they are magnetic in nature. While up and down DOS of Pd is symmetric in nature giving a total magnetic moment nearly zero, due to which it is non-magnetic in nature.

The magnetic moments for Fe, FePd and Fe3Pd are found to be $2.19 \mu_{\mathrm{B}}, 3.49 \mu_{\mathrm{B}}$ and $3.01 \mu_{\mathrm{B}}$ respectively, which are comparable to experimentally calculated value.

\section{Acknowledgments}

GCK is grateful to ICTP/OEA NET-56 program of Trieste, Italy for financial support and Prof. A. Mookerjee of S. N. Bose National Center For Basic Sciences, Kolkata, India for providing computational code and some discussion in the manuscript.

\section{References}

[1] R. M. Martin, Electronic structure: Basic Theory and Practical Methods, Cambridge University Press, United Kingdom, 2004.

[2] N. W. Ashcroft and N. D. Mermin, Solid State Physics, Thomson Books, USA, Second Edition 1976.

[3] R. A. Stern, S. D Willoughby, J. M. MacLaren, J. Cui, Q. Pan, R.D. James, J. Applied Physics, 93 (2003) 8644. 
S. Pandey et al. / BIBECHANA 11(1) (2014) 60-69: (Online Publication: March, 2014) p.69

[4] S. D Willoughby, J. M. MacLaren, Journal of Applied Physics, 91 (2002) 8813.

[5] P. Hohenberg, W. Kohn, Phys. Rev. B, 136 (1964) 864.

[6] U. V. Barth , L. Hedin, J. Phys. C : Solid State Phys, 5 (1972) 1629.

[7] H. L. Skriver, The LMTO Method: muffin-tin orbitals and electronic structure, SpringerVerlag, 1984.

[8] J. C. Slater and G. F. Koster. Phys. Rev. B, 15 (1954) 1498.

[9] W. Kohn, N. Rostoker, Phys. Rev., 94 (1954) 1111.

[10] O. K. Andersen, Phys. Rev. B, 12 (1975) 3060.

[11] O. K. Andersen, O. Jepsen, Phys. Rev Lett., 53 (1984) 2571.

[12] C. Kittel, Introduction to Solid State Physics, Wiley Publication, Seventh Edition 2009.

[13] A. Szabo, Neil S. Ostlund, Modern Quantum Chemistry, Dover Publication Inc., Second Edition 1996.

[14] S. Hollos, Electronic Band Structure Calculations, Exstrom Laboratories LLC, 1998.

[15] B. K. Aggrawal, H. Prakash, Quantum Mechanics, Prentice Hall Publication, 2007.

[16] W. Zohng, G. Overny, D. Tomanek, Phys. Rev. B, 47 (1993) 95. 